\title{
Adaptive support ventilation for faster weaning in COPD: a randomised controlled
} trial

\author{
C. Kirakli*, I. Ozdemir" ${ }^{\#}$ Z.Z. Ucar*, P. Cimen*, S. Kepil ${ }^{\#}$ and S.A. Ozkan"
}

ABSTRACT: Adaptive support ventilation (ASV) is a closed-loop ventilation mode that can act both as pressure support ventilation (PSV) and pressure-controlled ventilation. Weaning with ASV shows promising results, mainly in post-cardiac surgery patients. The aim of the present randomised controlled study was to test the hypothesis that weaning with ASV could reduce the weaning duration in patients with chronic obstructive pulmonary disease (COPD) when compared with PSV.

From among 435 COPD patients admitted to the intensive care unit (ICU) during a 20-month period, 97 were enrolled. Patients were assigned at random to either ASV or PSV as a weaning mode.

Compared with PSV, ASV provided shorter weaning times (median 24 (interquartile range 20-62) $h$ versus 72 (24-144) $h, p=0.041$ ) with similar weaning success rates (35 out of 49 for ASV and 33 out of 48 for PSV). Length of stay in the ICU was also shorter with ASV but the difference was not statistically significant.

This study suggests that ASV may be used in the weaning of COPD patients with the advantage of shorter weaning times. Further studies are needed to investigate the role and potential advantages of ASV in the weaning period of different patient groups.

KEYWORDS: Chronic obstructive pulmonary disease, intensive care unit, mechanical ventilation, weaning

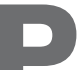

atients with acute exacerbations of chronic obstructive pulmonary disease (COPD) often require either noninvasive mechanical ventilation (NIMV) or invasive mechanical ventilation (IMV) and prolonged weaning times [1]. Although rapid weaning is preferable, there is still debate on the best weaning procedure [2]. Spontaneous breathing trials (SBT) with T-piece or pressure support ventilation (PSV) are common methods of weaning, but both require close patient observation for indicators of possible failure. Moreover, intensive care unit (ICU) staff experience with PSV is necessary to set the appropriate level of pressure [2-4]. Closed-loop modes and automated weaning procedures aim to set the most appropriate supporting pressure levels for the patient and promote early extubation, with conflicting results [5-8].

Adaptive support ventilation (ASV) is an improved closed-loop ventilation mode that provides both pressure-controlled ventilation and PSV according to the patient's needs [9-12]. Some studies have evaluated the use of ASV in weaning cardiac surgery patients and have shown a reduction in weaning time, a reduced need for arterial blood gas (ABG) analyses, and fewer ventilator adjustments $[9,10,13,14]$. The use of ASV in patients with COPD has been described previously $[12,15]$, but only one study reported the use of ASV as a weaning mode for chronically ventilated patients, some of whom had COPD [16]. Our study was therefore designed to compare ASV with PSV in the weaning of COPD patients.

\section{MATERIALS AND METHODS}

\section{Patients}

This randomised, controlled, single-blinded study was conducted over a 20-month period in the respiratory ICU of a hospital specialising in pulmonary diseases and thoracic surgery (Izmir Dr Suat Seren Chest Diseases and Thoracic Surgery Education and Research Hospital, Izmir, Turkey). The hospital is the largest regional education and research hospital in the western part of Turkey and has an ICU of 30 beds equipped with both invasive and noninvasive ventilation facilities. The study was approved by

\section{AFFILIATIONS}

*Intensive Care Unit, and

\#Pulmonary Division, Izmir Dr Suat Seren Chest Diseases and Thoracic Surgery Education and Research

Hospital, Izmir, Turkey.

\section{CORRESPONDENCE}

C. Kirakli

Intensive Care Unit, Izmir Dr Suat Seren Chest Diseases and Thoracic Surgery Education and Research Hospital

1671 sok. No

$159 \mathrm{D}$

535530 Karsiyaka Izmir

Turkey

E-mail: ckirakli@hotmail.com

Received:

May 252011

Accepted after revision:

Feb 142011

First published online:

March 152011 
the local institutional review board, and written informed consent was obtained from the patient and/or next of kin.

COPD patients with a confirmed diagnosis according to the Global Initiative for Chronic Obstructive Lung Disease (GOLD) criteria were included in the study [17]. They were orally intubated or replaced with an endotracheal tube of $\geqslant 8 \mathrm{~mm}$ internal diameter (if the endotracheal tube was $<8 \mathrm{~mm}$ in diameter) in the ICU to minimise the negative effect of tube resistance, and ventilated with a microprocessor-controlled mechanical ventilator (Galileo GOLD; Hamilton Medical AG, Bonaduz, Switzerland) using an assisted volume-controlled ventilation mode. Initial settings were as follows: tidal volume $(V \mathrm{~T}) 8 \mathrm{~mL} \cdot \mathrm{kg}^{-1}$, back-up respiratory rate (RR) 12-15 breath$\mathrm{s} \cdot \mathrm{min}^{-1}$ and positive end-expiratory pressure (PEEP) $3-5 \mathrm{cmH}_{2} \mathrm{O}$. Inspiratory oxygen fraction $\left(\mathrm{FI}_{1} \mathrm{O}_{2}\right)$ was titrated to obtain an arterial oxygen saturation $\left(\mathrm{Sa}_{\mathrm{a}} \mathrm{O}_{2}\right)>90 \%$. All the patients were receiving standard medical therapy with nebulised bronchodilators (ipratropium bromide plus salbutamol), corticosteroids, theophylline and antibiotics if needed. Sedation was achieved with midazolam and/or fentanyl. If the ABG analysis showed improvement in respiratory parameters $\left(\mathrm{pH} \geqslant 7.32\right.$, arterial oxygen tension $\left(\mathrm{Pa}_{1} \mathrm{O}_{2}\right) / \mathrm{FI}, \mathrm{O}_{2}>150$ with an $\mathrm{FI}, \mathrm{O}_{2} \leqslant 40 \%$ ), sedation was stopped and the patients were reevaluated for potential weaning [18]. Randomisation was performed when patients were in a stable condition (normal mental status, no signs of anxiety, somnolence or dyspnoea, and no severe respiratory acidosis and/or hypoxaemia in $\mathrm{ABG}$ ) and were able to trigger the ventilator effectively. Patients were not included if they had severe cardiac or neurological disease,sepsis, IMV $<24 \mathrm{~h}$ and a tracheostomy (fig. 1). Patients who had NIMV before intubation were also not enrolled because most of them received NIMV support in the pulmonary ward or emergency room and were managed by the non-intensivist pulmonary physicians. These factors may lead to delayed ICU admissions and late intubation, thus affecting the weaning period.

\section{ASV description}

ASV provides automatic ventilation in which minute volume is controlled via a $V \mathrm{~T} / \mathrm{RR}$ combination based on respiratory mechanics. In patients unable to trigger a breath, the ventilator generates pressure-controlled breaths, automatically adjusting inspiratory pressure and timing to achieve the target $V \mathrm{~T}$ and RR. In patients who are able to trigger a breath, the ventilator generates pressure support breaths, automatically adjusting the level of support pressure to achieve the target $V \mathrm{~T}$, and delivers additional pressure-controlled breaths if the patient's $\mathrm{RR}$ is below the target RR. The target $V \mathrm{~T} / \mathrm{RR}$ combination is based on the equation by OTIS et al. [19], which determines an RR that minimises work of inspiration for a clinician-set minute volume, based on the time constant of the respiratory system. The time constant is estimated on a breath-by-breath basis by the expiratory time constant (RCexp) obtained from the expiratory flow-volume curve [20, 21].

\section{Weaning protocols}

The weaning protocols are summarised in figure 2. After randomisation, the assisted volume-controlled mode was stopped and the two weaning modes (ASV and PSV) were allocated randomly using sealed envelopes according to a list

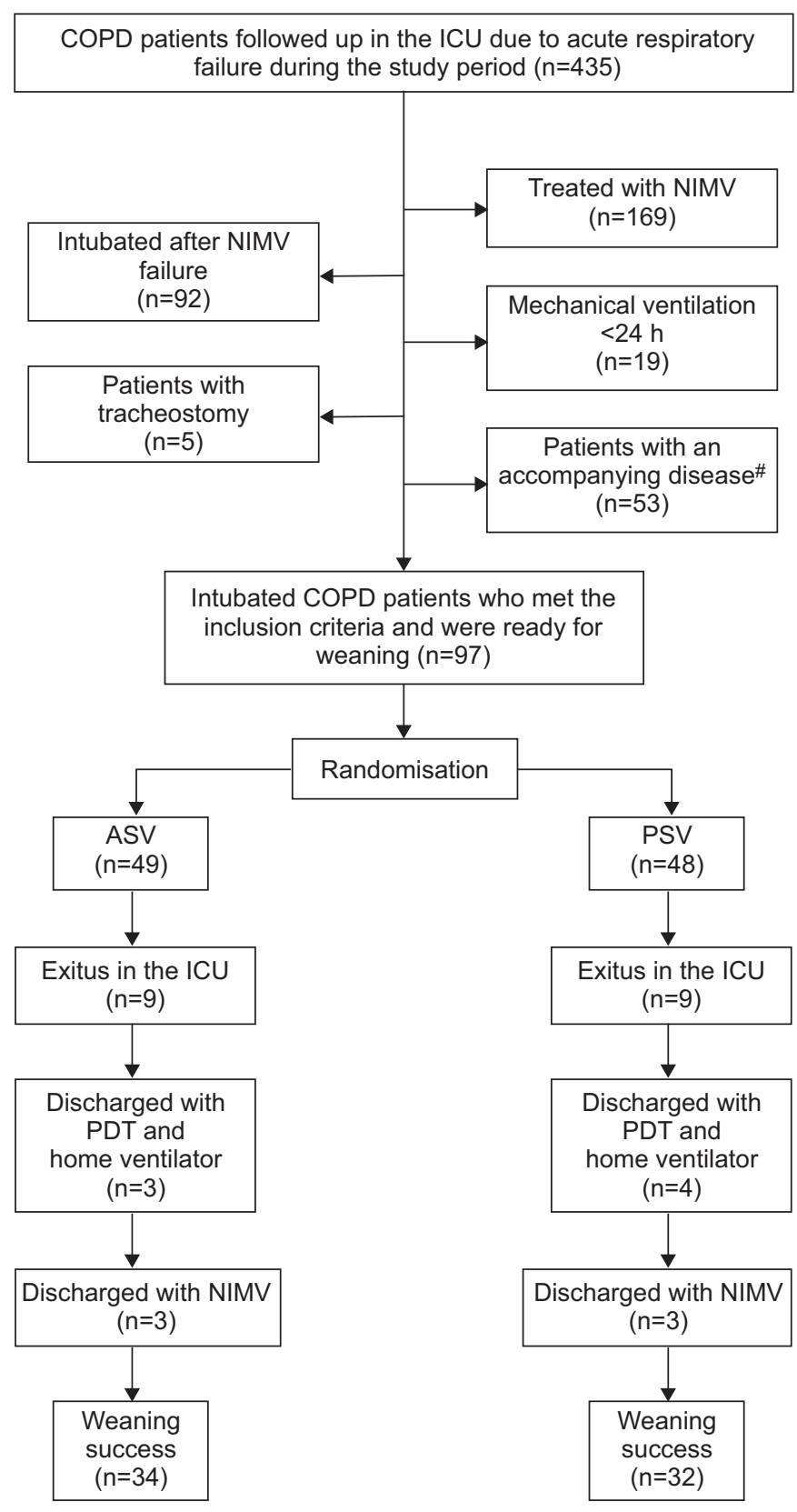

FIGURE 1. Flowchart of patients during the study. COPD: chronic obstructive pulmonary disease; ICU: intensive care unit; NIMV: noninvasive mechanical ventilation; ASV: adaptive support ventilation; PSV: pressure support ventilation; PDT: percutaneous dilatational tracheostomy. " : except cor pulmonale due to COPD.

of random numbers. Weaning and extubation were performed by the pulmonary and critical care physicians who were not aware of the study. In both modes of weaning, inspiratory trigger sensitivity was set at $-1 \mathrm{cmH}_{2} \mathrm{O}$. Pressure triggering was used to measure airway occlusion pressure at $0.1 \mathrm{~s}\left(P_{0.1}\right)$. High pressure alarm limit was set to $45 \mathrm{cmH}_{2} \mathrm{O}$ for ASV.

The initial level of pressure support (above PEEP) was set at $15 \mathrm{cmH}_{2} \mathrm{O}$ in the PSV group, as in the study of BURNS et al. [22]. The pressure support level was then evaluated at least every $30 \mathrm{~min}$ and titrated to keep the RR at $\leqslant 35$ breaths $\cdot \mathrm{min}^{-1}$ and, if possible, gradually decreased to $7 \mathrm{cmH}_{2} \mathrm{O}$ by $2 \mathrm{cmH}_{2} \mathrm{O}$ 


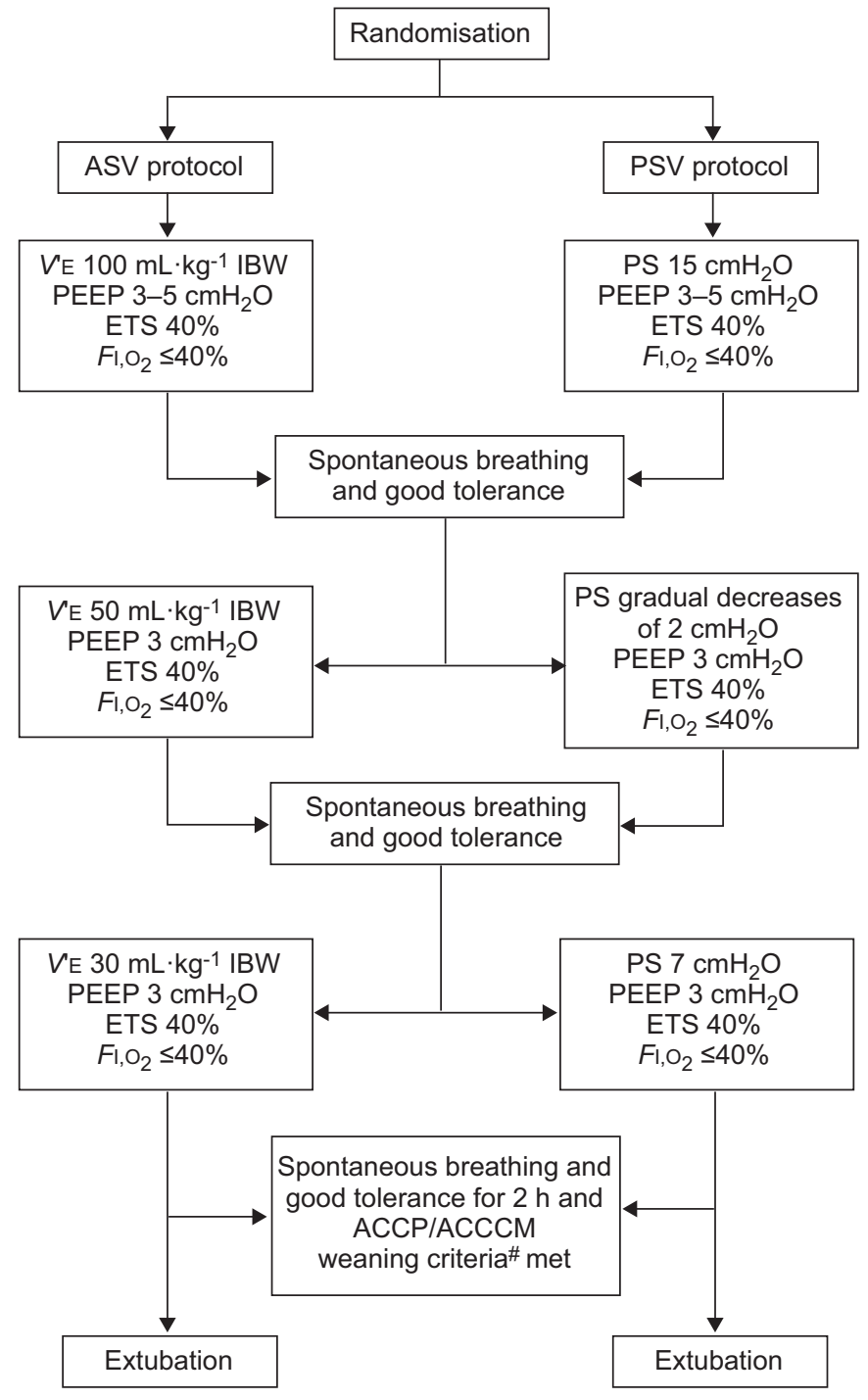

FIGURE 2. Weaning protocols for the adaptive support ventilation (ASV) group and the pressure support ventilation (PSV) group. V'E: minute ventilation; IBW: ideal body weight; PEEP: positive end-expiratory pressure; ETS: expiratory trigger sensitivity; $\mathrm{Fl}_{1} \mathrm{O}_{2}$ : inspiratory oxygen fraction; PS: pressure support; ACCP/ACCCM: American College of Chest Physicians/American College of Critical Care Medicine. \#: good tolerance criteria is clinical stability (haemodynamically stable, normal mental status, and no signs of anxiety, somnolence or dyspnoea) with an acceptable arterial blood gas analysis $\left(\mathrm{pH} \geqslant 7.35\right.$, arterial oxygen tension $/ \mathrm{Fl}, \mathrm{O}_{2}$ $>150$ with an $\mathrm{Fl}_{1} \mathrm{O}_{2} \leqslant 40 \%$, respiratory rate $\leqslant 35$ breaths $\cdot \mathrm{min}^{-1}$ ).

intervals. In patients in whom a $7 \mathrm{cmH}_{2} \mathrm{O}$ pressure support level could be achieved, a 2-h trial of spontaneous breathing with this pressure support level was performed before extubation [23].

In the ASV group, after randomisation, minute volume was set at $100 \mathrm{~mL} \cdot \mathrm{kg}^{-1}$ ideal body weight (IBW). As tolerated (haemodynamically stable, normal mental status, and no signs of anxiety, somnolence or dyspnoea), the minute volume was decreased to $50 \mathrm{~mL} \cdot \mathrm{kg}^{-1}$ IBW after $1 \mathrm{~h}$ and to $30 \mathrm{~mL} \cdot \mathrm{kg}^{-1}$ IBW after $2 \mathrm{~h}$, which would be predicted to be associated with an inspiratory pressure level $<10 \mathrm{cmH}_{2} \mathrm{O}$, similar to that with PSV. Patients underwent an SBT with $30 \mathrm{~mL} \cdot \mathrm{kg}^{-1}$ IBW minute volume support for $2 \mathrm{~h}$ before extubation, similar to that in PSV.
If the patients showed good tolerance with an acceptable ABG analysis $\left(\mathrm{pH} \geqslant 7.35, \mathrm{~Pa}_{1} \mathrm{O}_{2} / \mathrm{FI}_{1} \mathrm{O}_{2}>150\right.$ with an $\mathrm{FI}_{1} \mathrm{O}_{2} \leqslant 40 \%$, RR $\leqslant 35$ breaths $\cdot \mathrm{min}^{-1}$ ), they were ventilated with the above final settings for $2 \mathrm{~h}$ and then extubated. Otherwise, the trial was stopped and patients were ventilated with assisted volumecontrolled ventilation mode during the night. They were evaluated for weaning again the following day.

Post-extubation failure occurring within the first $48 \mathrm{~h}$ was defined as $\mathrm{pH} \leqslant 7.35$, an increase in arterial carbon dioxide tension $\geqslant 15 \mathrm{mmHg}$ from the value just prior to extubation, RR $>24$ breaths $\cdot \mathrm{min}^{-1}$ and accessory muscle use. NIMV trial with a full face mask was performed in these patients using the same ventilator in NIMV mode to avert re-intubation [1, 24, 25]. Patients who could not tolerate NIMV or showed impairment in their clinical status (unable to protect airway, inability to remove secretions, cardiac instability or loss of consciousness) or blood gas analysis $\left(\mathrm{pH} \leqslant 7.25\right.$ and $\mathrm{Pa}_{\mathrm{a}_{2}}<60 \mathrm{mmHg}$ while receiving NIMV) were re-intubated. These patients were considered as having failed weaning irrespective of their outcomes using NIMV.

\section{Data collection}

Demographic data, medical history, Acute Physiology and Chronic Health Evaluation (APACHE) II scores and duration of mechanical ventilation before randomisation were recorded. During the weaning period, the ventilator was connected to a personal computer via an RS232 serial port and data were recorded using data acquisition software (Hamilton Medical Ventilator Data Logger Version 3.27; Hamilton Medical AG). Breath-by-breath $V \mathrm{~T}$, minute volume, RCexp, $P_{0.1}$, inspiratory pressure support level and RR directly obtained from the digital readout of the ventilator and ABG measurements were recorded. Only the values from the last $15 \mathrm{~min}$ of the successful 2-h period before extubation were averaged and analysed.

\section{Outcomes and definitions}

Weaning duration was the primary outcome, defined as the time from randomisation to spontaneous breathing with or without a tracheostomy. Weaning success was defined as independence from mechanical ventilation (invasive or noninvasive) $\geqslant 48 \mathrm{~h}$ after extubation or with a tracheostomy cannula at day 28. Secondary outcomes were weaning success rates, respiratory parameters at the end of the weaning period, duration of mechanical ventilation and length of stay (LOS) in the ICU.

Duration of mechanical ventilation was defined as the time from the initiation of mechanical ventilation support to the permanent cessation of any form of ventilatory support (invasive or noninvasive). Duration of IMV before was defined as the time of IMV from intubation to the time of randomisation. LOS in the ICU was defined as the time from admission to the ICU until discharge or death.

\section{Statistical analysis}

The sample size of a minimum of 45 in each group was chosen to give a power of 0.80 in order to detect a 6 -h reduction in mean weaning time, assuming an SD of $10 \mathrm{~h}$ with a two-sided test at the 0.05 level. Statistical analyses was performed with Statistica 8.0 software (Statsoft Inc., Tulsa, OK, USA). Data are expressed as median (interquartile range (IQR)). Weaning durations were compared by log rank test. Other comparisons 
TABLE 1 Demographic and respiratory data before randomisation

\begin{tabular}{|c|c|c|c|}
\hline Subjects $n$ & 49 & 48 & \\
\hline Males & $44(90)$ & $45(93)$ & 0.36 \\
\hline BMI $\mathbf{~ k g} \cdot \mathrm{cm}^{-2}$ & $25(21-27)$ & $25(22-29)$ & 0.23 \\
\hline APACHE II & $16(14-19)$ & $16(14-19)$ & 0.74 \\
\hline $\mathrm{HCO}_{3} \mathrm{mmol} \cdot \mathrm{L}^{-1}$ & $36(31-39)$ & $36(29-40)$ & 0.88 \\
\hline $\mathrm{Pa}, \mathrm{O}_{2} / \mathrm{Fl}_{1} \mathrm{O}_{2}$ & $182(170-198)$ & $176(168-184)$ & 0.23 \\
\hline Duration of prior invasive MV h & $48(48-96)$ & $67(48-96)$ & 0.82 \\
\hline Patients needing sedation before randomisation & $16(32)$ & $15(31)$ & 0.52 \\
\hline
\end{tabular}

Data are presented as median (interquartile range) or $\mathrm{n}(\%)$, unless otherwise stated. ASV: adaptive support ventilation; PSV: pressure support ventilation; BMI: body mass index; APACHE: Acute Physiology and Chronic Health Evaluation; $\mathrm{Pa}_{1} \mathrm{CO}_{2}$ : arterial carbon dioxide tension; $\mathrm{Pa}, \mathrm{O}_{2} / \mathrm{Fl}, \mathrm{O} 2$ : arterial oxygen tension/inspiratory oxygen fraction; MV: mechanical ventilation.

between groups were performed by nonparametric MannWhitney U-test. Differences in proportions between groups were evaluated using Fischer's exact test. A p-value of $<0.05$ was considered significant.

\section{RESULTS}

During the study period, 4,228 out of 15,099 hospitalised patients had a diagnosis of COPD, of which 435 were admitted to the ICU because of acute respiratory failure. Of the 435 patients, 261 were treated with NIMV as a first-line treatment and 92 of them subsequently required intubation (fig. 1).

The ASV and PSV groups were demographically similar at the time of randomisation. Factors that could affect weaning, such as the severity of patients assessed by APACHE II score, need of sedation and respiratory parameters, were also comparable between the two groups (table 1).

79 patients were extubated after the weaning period. Respiratory data during the final $15 \mathrm{~min}$ of the weaning period before extubation are shown in table 2. 14 patients in the ASV group (28\%) and 15 patients in the PSV group (31\%) were considered to be weaning failures $(p>0.05)$. Three patients in the ASV group and four in the PSV group could not tolerate extubation and underwent a percutaneous dilatational tracheostomy procedure. These patients also could not be weaned and were discharged with a home ventilator. Two patients in the ASV group and three in the PSV group were also discharged from the ICU under NIMV support. Weaning duration was significantly shorter with ASV versus PSV (median (IQR) 24 (20-62) h versus 72 (24-144) h, p=0.041). Weaning duration for the two groups expressed as KaplanMeier curves are shown in figure 3. LOS in the ICU WAS also shorter with ASV compared with PSV (11 (6-15) days versus 13 (8-14) days, $p=0.5$ ) (table 3) but this difference was not statistically significant.

\section{DISCUSSION}

The major finding of this study was that, when compared with PSV in the weaning process of COPD patients, ASV was associated with a shorter weaning duration.

\section{Weaning duration}

Of the few studies evaluating ASV in weaning, most were performed in post-cardiac surgery patients where the mean extubation time was $<6 \mathrm{~h}$. No systematic data were collected in COPD patients, where the duration of the weaning process is usually much longer and the weaning process is much more complicated. Sulzer et al. [9] reported shorter duration of intubation and mechanical ventilation with ASV than

\section{TABLE 2 Monitored respiratory data and arterial blood gases of extubated patients during the last 15 min of the weaning period}

\begin{tabular}{|c|c|c|c|}
\hline & ASV & PSV & $\mathrm{p}$-value \\
\hline Subjects $\mathrm{n}$ & 40 & 39 & \\
\hline PS level & $7(6-8)$ & $7(7-7)$ & 0.12 \\
\hline $\mathrm{pH}$ & $7.41(7.35-7.45)$ & $7.39(7.33-7.45)$ & 0.54 \\
\hline $\mathrm{Pa}, \mathrm{CO}_{2} \mathrm{mmHg}$ & $56(51-64)$ & $57(50-66)$ & 0.62 \\
\hline $\mathrm{HCO}_{3} \mathrm{mmol} \cdot \mathrm{L}^{-1}$ & $36(35-40)$ & $36(29-40)$ & 0.6 \\
\hline $\mathrm{Pa}, \mathrm{O}_{2} / \mathrm{Fl}_{1} \mathrm{O}_{2}$ & $288(230-310)$ & 267 (230-297) & 0.46 \\
\hline$V^{\prime} E L \cdot \min ^{-1}$ & $8.3(7.4-9.1)$ & $8.4(6.9-9.8)$ & 0.87 \\
\hline$V^{\prime} E \mathrm{~mL} \cdot \mathbf{k g}^{-1}$ & $119(101-148)$ & $126(103-133)$ & 0.91 \\
\hline VT $\mathrm{mL}$ & 394 (333-489) & $424(326-474)$ & 0.74 \\
\hline$V_{T} \mathbf{m L} \cdot \mathbf{k g}^{-1}$ & $5.7(5.3-6.3)$ & $5.4(4.9-6.9)$ & 0.45 \\
\hline $\begin{array}{c}\text { Respiratory rate } \\
\text { breaths } \cdot \mathrm{min}^{-1}\end{array}$ & $22(17-26)$ & $23(20-27)$ & 0.43 \\
\hline $\mathbf{R C} \exp \mathbf{s}$ & $0.9(0.7-1.1)$ & $0.8(0.7-0.9)$ & 0.37 \\
\hline tE S & $1.9(1.5-2.5)$ & $1.7(1.4-2)$ & 0.16 \\
\hline$t \mid s$ & $0.8(0.7-1.2)$ & $0.9(0.8-1)$ & 0.81 \\
\hline$P_{0.1} \mathrm{cmH}_{2} \mathrm{O}$ & $2.8(1.1-3.9)$ & $2.3(2-3.4)$ & 0.57 \\
\hline
\end{tabular}

Data are presented as median (interquartile range), unless otherwise stated. ASV: adaptive support ventilation; PSV: pressure support ventilation; PS pressure support; $\mathrm{Pa}, \mathrm{CO}_{2}$ : arterial carbon dioxide tension; $\mathrm{Pa}, \mathrm{O}_{2} / \mathrm{FI}_{1} \mathrm{O}_{2}$ : arterial oxygen tension/inspiratory oxygen fraction; $V^{\prime} E$ : minute ventilation; $V T$ : tidal volume; RCexp: expiratory time constant; $t \mathrm{E}$ : expiratory time; tl: inspiratory time; P0.1: airway occlusion pressure at $0.1 \mathrm{~s}$. 


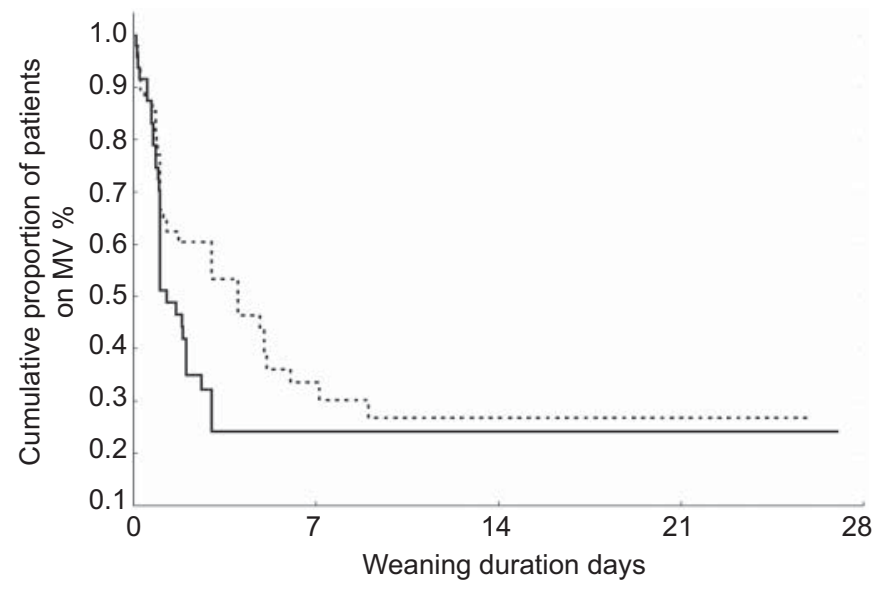

FIGURE 3. Duration of weaning expressed as a Kaplan-Meier curve in the adaptive support ventilation $(-)$ and pressure support ventilation $(\cdots \cdots \cdot)$ groups. MV: mechanical ventilation. Log rank test, $p=0.15$.

synchronised intermittent mandatory ventilation (SIMV) in post-operative coronary bypass patients. CASSINA et al. [5] used ASV when weaning 155 cardiac surgery patients; 86\% were extubated within $6 \mathrm{~h}$ and the mean time to extubation was $3.6 \mathrm{~h}$. In a randomised controlled trial, PETTER et al. [10] compared ASV with SIMV+PSV when weaning 45 cardiac surgery patients and found that the duration of mechanical ventilation and the need for changing the ventilator settings were reduced with ASV. These findings suggest that ASV could be used for fast and early extubation after post-cardiac surgery. However, none of these studies included COPD patients.

In our study, the median weaning duration in ASV and PSV was 24 and $72 \mathrm{~h}$, respectively, whereas in a similar population, MATIC and MAJERIC-KOGLER [26] reported a weaning time of $54 \mathrm{~h}$ with PSV. Weaning times with PSV have been reported up to $115 \mathrm{~h}$ [27]. In a recent study, weaning times with ASV and PSV were comparable (16.4 $\mathrm{h}$ and $16.3 \mathrm{~h}$, respectively); however, the study was performed in cardiothoracic surgery patients with normal lungs [13]. The shorter weaning times with ASV in our study might be due to the automation of inspiratory pressure levels and reduced manipulation and time spent adjusting the ventilator (manually in PSV versus automatic in ASV). Some data suggest that the automation of inspiratory pressures with a computer-driven system may lead the patients to spend much more time in the comfort zone of ventilation [28]. Increased patient ventilator interaction and decreased impact of the ICU staff in the setting of appropriate pressure support levels for each individual patient in ASV may be the potential reason for faster weaning.

It is obvious that shorter duration of intubation will result in shorter LOS in the ICU. NIMV seems to be an effective means of early extubation and reduction of the ICU LOS, especially in COPD patients [29]. ICU stay was also shorter in the ASV group but the effect of this closed-loop mode as a weaning technique on ICU stay still remains uncertain. Automated closed-loop ventilation modes may be promising alternatives for conventional weaning techniques.

\begin{tabular}{|c|c|c|c|c|}
\hline \multirow{2}{*}{$\begin{array}{l}\text { TABLE } 3 \\
\text { Outcomes }\end{array}$} & \multicolumn{4}{|c|}{$\begin{array}{l}\text { Comparison of adaptive support ventilation } \\
\text { (ASV) and the pressure support ventilation (PSV) } \\
\text { groups }\end{array}$} \\
\hline & & ASV & PSV & p-value \\
\hline \multicolumn{2}{|l|}{ Subjects $n$} & 49 & 48 & \\
\hline \multicolumn{2}{|c|}{ Weaning duration $\mathrm{h}$} & $24(20-62)$ & $72(24-169)$ & 0.041 \\
\hline \multicolumn{2}{|l|}{ Weaning failure } & $15(31)$ & $16(33)$ & 0.47 \\
\hline \multicolumn{2}{|c|}{ Duration of $\mathrm{MV} \mathbf{h}$} & $120(72-264)$ & 156 (72-288) & 0.56 \\
\hline \multicolumn{2}{|c|}{ LOS in ICU days } & $11(6-15)$ & $13(8-14)$ & 0.5 \\
\hline \multicolumn{2}{|c|}{ Mortality at day 28} & $9(18)$ & $9(18)$ & 0.58 \\
\hline \multicolumn{5}{|c|}{$\begin{array}{l}\text { Data are presented as median (interquartile range) or } n(\%) \text {, unless otherwise } \\
\text { stated. MV: mechanical ventilation; LOS: length of stay; ICU: intensive care unit. } \\
\text { Bold indicates statistical significance. }\end{array}$} \\
\hline
\end{tabular}

\section{Weaning protocols and settings}

Randomised controlled studies and subsequent meta-analysis indicated that SBT with T-piece or PSV are equally effective and both superior to SIMV, depending in the most part on the experience of the staff with a particular method [30-33]. TASSAUX et al. [12] compared ASV and SIMV+PSV patientventilator interactions in 10 patients, three of whom had acute exacerbations of COPD; they concluded that ASV could provide the same minute ventilation with less muscle load and patient-ventilator dyssynchrony when compared with SIMV+PSV. In addition, similar levels of minute ventilation and $V \mathrm{~T}(\mathrm{~mL}) /$ patients $(\mathrm{kg})$ ratio were achieved with ASV.

Pressure support levels were set between 5 and $10 \mathrm{cmH}_{2} \mathrm{O}$ in studies of weaning with PSV; such levels were suggested to be effective in overcoming the resistance and workload of endotracheal tube and respiratory circuits [26, 34-37]. SULZER et al. [9] decreased minute volume to $25 \%$ of the baseline value in cardiac surgery patients and achieved extubation with this level of support. LINTON et al. [16] decreased minute volume to $60 \%$ before extubation in chronically ventilated patients. In our study, minute volume was decreased to $30 \%$ in the ASV mode to achieve lower pressure support levels, as in the PSV group, and to prepare the patient for extubation. However, after analysis of the data, we detected that some patients had pressure support levels $>7 \mathrm{cmH}_{2} \mathrm{O}$ in the ASV group, although the median pressure support levels of PSV and ASV were comparable. The ICU staff in charge of the weaning process made the extubation decision according to the American College of Chest Physicians/ American College of Critical Care Medicine weaning and extubation criteria, without taking into account the pressure support levels in the ASV group. This might have caused the inappropriate extubation of some patients in the ASV group. Bedside monitoring of the pressure support levels during weaning with ASV may lead to better prediction of weaning outcome.

\section{Predictors and success rates of ASV and PSV weaning}

The threshold values of different weaning indices and measured respiratory parameters obtained in COPD patients could differ from those in nonhomogeneous populations. ALVISI et al. [38] found threshold values for minute volume, $V \mathrm{~T}$ and $V \mathrm{~T}(\mathrm{~mL}) /$ patients $(\mathrm{kg})$ ratio for weaning success in COPD patients to be 
8.6 $\mathrm{L} \cdot \mathrm{min}^{-1}, 340 \mathrm{~mL}$ and $5.0 \mathrm{~mL} \cdot \mathrm{kg}^{-1}$, respectively. In the present study, median values for all these parameters in both groups were found to be in the appropriate range for successful weaning, and there was no significant difference between ASV and PSV when these data were compared. In addition, P0.1, another index of respiratory drive, showed no significant difference between ASV and PSV and was found to be $<6 \mathrm{cmH}_{2} \mathrm{O}$, which was suggested by MANTHOus et al. [39] as the successful weaning threshold value for COPD patients.

ESTEBAN et al. [23] reported weaning success rates of $70 \%$ for PSV and $63 \%$ for SBT. Their study was performed on a heterogeneous population, but $20 \%$ of the patients enrolled had COPD. Our study found weaning success rates of $69 \%$ for each group, similar to those in the literature.

\section{Limitations of the study}

Some limitations of the study are worth noting. Concerning the study design, in the PSV group, the absolute pressure support level the patients were receiving was $7 \mathrm{cmH}_{2} \mathrm{O}$, which is accepted to be as effective as an SBT with a T-piece [2]. However, in the ASV group, some patients who had weaning failure had pressure support levels $>10 \mathrm{cmH}_{2} \mathrm{O}$, even up to $18 \mathrm{cmH}_{2} \mathrm{O}$, although the median PS levels were comparable. Taking into account the pressure support levels during the weaning period, while using ASV as a weaning mode, might be a good predictor of weaning outcome, particularly in COPD patients.

We randomly allocated the patients into two modes when they were ready for weaning. Some studies concerning the weaning procedures suggested that SBT's with T-piece were superior to SIMV and PSV [32], while others showed that gradual reduction of pressure support was superior in patients who failed a 2-h SBT with T-piece [30]. Further studies are needed to assess the feasibility of ASV in difficult to wean patients who fail SBTs with a T-piece. Another interesting area may be the use of closed-loop modes from the beginning of intubation and mechanical ventilation until the end of weaning and comparison of the total duration of ventilation and LOS in the ICU with the conventional modes and weaning techniques. We used assisted volume-controlled ventilation mode until randomisation and on night shifts during the weaning period in order to avoid fatigue and prepare the patient for the weaning trial the next day. This might have caused a bias and underestimation of the potential benefits of ASV on total ventilation time and ICU stay because the total time patients spent on assisted volume-controlled ventilation was not evaluated.

Patients with previous use of NIV were not included in the study. The reason for this was to evaluate the effects of these modes in a more homogenous group. However, in real-world situations, most patients with COPD are intubated after failure of NIV. This limits the generalisation of the results to all intubated patients with COPD.

This single centre study reflects the skills and experience of a single unit. With 49 patients in the ASV group and 48 in the PSV groups to detect a reduction of 2 days in the weaning time assuming an SD of 4 days, our study seemed to have a power of 0.68 . Multicentric, higher powered studies with large sample sizes could more accurately assess the generalisability of these results to different centres and patient populations. The lack of
ICU staff prevented us from recording the number of interventions and ABG sampling needed for each group. Another potentially interesting area might be the comparison of patient comfort and respiratory mechanics, such as work of breathing and pressure time product in ASV with different weaning modes. Further studies are needed to determine the advantages of this closed-loop automated ventilation mode.

\section{Conclusions}

The results of our study suggest that ASV may be used as a weaning mode in severe COPD patients, with the advantage of shorter weaning duration. Further studies with large sample sizes are needed to investigate the role and potential advantages of this closed-loop ventilation mode in the weaning period and ICU stay of different patient groups.

\section{STATEMENT OF INTEREST}

None declared.

\section{ACKNOWLEDGEMENTS}

We wish to thank those involved in the American Thoracic Society's MECOR (Methods in Epidemiologic, Clinical and Operations Research) program, and P. Hopewell (Pulmonary and Critical Care Division, San Francisco General Hospital, University of California San Francisco, San Francisco, CA, USA) in particular, for stimulating interest in research and providing advice about this paper. Professional editing of this paper was funded by MECOR.

\section{REFERENCES}

1 Nava S, Hill N. Non-invasive ventilation in acute respiratory failure. Lancet 2009; 374: 250-259.

2 Boles JM, Bion J, Connors A, et al. Weaning from mechanical ventilation. Eur Respir J 2007; 29: 1033-1056.

3 Brochard L, Harf A, Lorino $\mathrm{H}$, et al. Inspiratory pressure support prevents diaphragmatic fatigue during weaning from mechanical ventilation. Am Rev Respir Dis 1989; 139: 513-521.

4 Esteban A, Anzueto A, Frutos F, et al. Characteristics and outcomes in adult patients receiving mechanical ventilation: a 28-day international study. JAMA 2002; 287: 345-355.

5 Cassina T, Chiolero R, Mauri R, et al. Clinical experience with adaptive support ventilation for fast-track cardiac surgery. J Cardiothorac Vasc Anesth 2003; 17: 571-575.

6 Bouadma L, Lellouche F, Cabello B, et al. Computer-driven management of prolonged mechanical ventilation and weaning: a pilot study. Intensive Care Med 2005; 31: 1446-1450.

7 Rose L, Presneill JJ, Johnston L, et al. A randomised, controlled trial of conventional versus automated weaning from mechanical ventilation using SmartCare/PS. Intensive Care Med 2008; 34: 1788-1795.

8 Lellouche F, Mancebo J, Jolliet P, et al. A multicenter randomized trial of computer-driven protocolized weaning from mechanical ventilation. Am J Respir Crit Care Med 2006; 174: 894-900.

9 Sulzer CF, Chiolero R, Chassot PG, et al. Adaptive support ventilation for fast tracheal extubation after cardiac surgery: a randomized controlled study. Anesthesiology 2001; 95: 1339-1345.

10 Petter AH, Chiolero RL, Cassina T, et al. Automatic "respirator/ weaning" with adaptive support ventilation: the effect on duration of endotracheal intubation and patient management. Anesth Analg 2003; 97: 1743-1750.

11 Hamilton Medical AG. Adaptive Support Ventilation User' Guide. Bonaduz, Hamilton Medical, 1999.

12 Tassaux D, Dalmas E, Gratadour P, et al. Patient-ventilator interactions during partial ventilatory support: a preliminary study comparing the effects of adaptive support ventilation with 
synchronized intermittent mandatory ventilation plus inspiratory pressure support. Crit Care Med 2002; 30: 801-807.

13 Dongelmans DA, Veelo DP, Paulus F, et al. Weaning automation with adaptive support ventilation: a randomized controlled trial in cardiothoracic surgery patients. Anesth Analg 2009; 108: 565-571.

14 Gruber PC, Gomersall CD, Leung P, et al. Randomized controlled trial comparing adaptive-support ventilation with pressure-regulated volume-controlled ventilation with automode in weaning patients after cardiac surgery. Anesthesiology 2008; 109: 81-87.

15 Arnal JM, Wysocki M, Nafati C, et al. Automatic selection of breathing pattern using adaptive support ventilation. Intensive Care Med 2008; 34: 75-81.

16 Linton DM, Renov G, Lafair J, et al. Adaptive support ventilation as the sole mode of ventilatory support in chronically ventilated patients. Crit Care Resusc 2006; 8: 11-14.

17 Asia-Pacific COPD Roundtable Group. Global Initiative for Chronic Obstructive Lung Disease strategy for the diagnosis, management and prevention of chronic obstructive pulmonary disease: an Asia-Pacific perspective. Respirology 2005; 10: 9-17.

18 MacIntyre NR, Cook DJ, Ely EW Jr, et al. Evidence-based guidelines for weaning and discontinuing ventilatory support: a collective task force facilitated by the American College of Chest Physicians, the American Association for Respiratory Care, and the American College of Critical Care Medicine. Chest 2001; 120 : Suppl. 6, 375S-395S.

19 Otis AB, Fenn WO, Rahn H. Mechanics of breathing in man. J Appl Physiol 1950; 2: 592-607.

20 Brunner JX, Laubscher TP, Banner MJ, et al. Simple method to measure total expiratory time constant based on the passive expiratory flow-volume curve. Crit Care Med 1995; 23: 1117-1122.

21 Lourens MS, van den Berg B, Aerts JG, et al. Expiratory time constants in mechanically ventilated patients with and without COPD. Intensive Care Med 2000; 26: 1612-1618.

22 Burns KE, Meade MO, Lessard MR, et al. Wean earlier and automatically with new technology (the WEAN study): a protocol of a multicentre, pilot randomized controlled trial. Trials 2009; 10: 81.

23 Esteban A, Alia I, Gordo F, et al. Extubation outcome after spontaneous breathing trials with T-tube or pressure support ventilation. The Spanish Lung Failure Collaborative Group. Am J Respir Crit Care Med 1997; 156: 459-465.

24 Hilbert G, Gruson D, Portel L, et al. Noninvasive pressure support ventilation in COPD patients with postextubation hypercapnic respiratory insufficiency. Eur Respir J 1998; 11: 1349-1353.

25 Ferrer M, Valencia M, Nicolas JM, et al. Bernadich early noninvasive ventilation averts extubation failure in patients at risk: a randomized trial. Am J Respir Crit Care Med 2006; 173: 164-170.

26 Matic I, Majeric-Kogler V. Comparison of pressure support and T-tube weaning from mechanical ventilation: randomized prospective study. Croat Med J 2004; 45: 162-166.

27 Esteban A, Alia I, Ibanez J, et al. Modes of mechanical ventilation and weaning. A national survey of Spanish hospitals. The Spanish Lung Failure Collaborative Group. Chest 1994; 106: 1188-1193.

28 Dojat M, Harf A, Touchard D, et al. Clinical evaluation of a computer-controlled pressure support mode. Am J Respir Crit Care Med 2000; 161: 1161-1166.

29 Nava S, Ambrosino N, Clini E, et al. Noninvasive mechanical ventilation in the weaning of patients with respiratory failure due to chronic obstructive pulmonary disease. A randomized, controlled trial. Ann Intern Med 1998; 128: 721-728.

30 Brochard L, Rauss A, Benito S, et al. Comparison of three methods of gradual withdrawal from ventilatory support during weaning from mechanical ventilation. Am J Respir Crit Care Med 1994; 150: 896-903.

31 Vitacca M, Vianello A, Colombo D, et al. Comparison of two methods for weaning patients with chronic obstructive pulmonary disease requiring mechanical ventilation for more than 15 days. Am J Respir Crit Care Med 2001; 164: 225-230.

32 Esteban A, Frutos F, Tobin MJ, et al. A comparison of four methods of weaning patients from mechanical ventilation. Spanish Lung Failure Collaborative Group. N Engl J Med 1995; 332: 345-350.

33 Hess D. Ventilator modes used in weaning. Chest 2001; 120: Suppl. 6, 474S-476S.

34 Jubran A, Van de Graaff WB, Tobin MJ. Variability of patientventilator interaction with pressure support ventilation in patients with chronic obstructive pulmonary disease. Am J Respir Crit Care Med 1995; 152: 129-136.

35 Fiastro JF, Habib MP, Quan SF. Pressure support compensation for inspiratory work due to endotracheal tubes and demand continuous positive airway pressure. Chest 1988; 93: 499-505.

36 Brochard L, Rua F, Lorino $\mathrm{H}$, et al. Inspiratory pressure support compensates for the additional work of breathing caused by the endotracheal tube. Anesthesiology 1991; 75: 739-745.

37 Kollef MH, Shapiro SD, Silver P, et al. A randomized, controlled trial of protocol-directed versus physician-directed weaning from mechanical ventilation. Crit Care Med 1997; 25: 567-574.

38 Alvisi R, Volta CA, Righini ER, et al. Predictors of weaning outcome in chronic obstructive pulmonary disease patients. Eur Respir J 2000; 15: 656-662.

39 Manthous CA, Schmidt GA, Hall JB. Liberation from mechanical ventilation: a decade of progress. Chest 1998; 114: 886-901. 